\title{
Exact Multiplicity of Solutions for a Class of Singular Generalized One-Dimensional $p$-Laplacian Problem
}

\author{
Youwei Zhang \\ Department of Mathematics, Hexi University, Gansu 734000, China \\ Correspondence should be addressed to Youwei Zhang; ywzhang0288@163.com
}

Received 11 November 2012; Accepted 29 May 2013

Academic Editor: Francisco B. Gallego

Copyright (C) 2013 Youwei Zhang. This is an open access article distributed under the Creative Commons Attribution License, which permits unrestricted use, distribution, and reproduction in any medium, provided the original work is properly cited.

We describe the existence of positive solutions for a class of singular generalized one-dimensional $p$-Laplacian problem. By applying the related fixed point theory in cone, some new and general results on the existence of positive solutions to the singular generalized $p$-Laplacian problem are obtained. Note that the nonlinear term $f$ involves the first-order derivative explicitly.

\section{Introduction}

Recently, increasing attention is paid to question of positive solution for singular boundary value problems [1-11]. One notices that the singular boundary value problems for ordinary differential equation describe many phenomena in applied mathematics and physical science, which can be found in the theory of nonlinear diffusion generated by nonlinear sources and in the thermal ignition of gases [12-17]. Moreover, there are excellent results of nonsingular problem, see $[18-21]$ and the references therein.

Moreover, the nonlocal $p$-Laplacian problems for ordinary differential equation have been studied extensively. There are many papers dealing with the existence of positive solutions for the nonlocal $p$-Laplacian boundary value problem, in which the nonlinear term $f$ is independent of the first-order derivative with different boundary conditions. For example, Ma et al. [22] established some existence results of positive solutions for the problem

$$
\begin{aligned}
& \left(\varphi_{p}\left(u^{\prime}\right)\right)^{\prime}+q(t) f(t, u)=0, \quad t \in(0,1), \\
& u^{\prime}(0)=\sum_{i=1}^{m-2} \alpha_{i} u^{\prime}\left(\xi_{i}\right), \quad u(1)=\sum_{i=1}^{m-2} \beta_{i} u\left(\xi_{i}\right) .
\end{aligned}
$$

The main tool is the monotone iterative technique. By applications of fixed point index theory, Cheung and
Ren [23] investigated two classes of quasilinear nonlocal problems:

$$
\begin{gathered}
\left(\varphi_{p}\left(u^{\prime}\right)\right)^{\prime}+h(t) f(t, u)=0, \quad 0<t<1, \\
u^{\prime}(0)=0, \quad u(1)=\sum_{i=1}^{m-2} \alpha_{i} u\left(\xi_{i}\right), \\
u(0)=\sum_{i=1}^{m-2} \alpha_{i} u\left(\xi_{i}\right), \quad u^{\prime}(1)=0 .
\end{gathered}
$$

Sufficient conditions for the existence of twin positive solutions are established. In [16], Feng and Ge studied nonlocal problem for the one-dimensional $p$-Laplacian

$$
\begin{gathered}
\left(\varphi_{p}\left(u^{\prime}\right)\right)^{\prime}+q(t) f\left(t, u, u^{\prime}\right)=0, \quad t \in(0,1), \\
u(0)=0, \quad u(1)=\sum_{i=1}^{m-2} a_{i} u\left(\xi_{i}\right) .
\end{gathered}
$$

They obtained sufficient conditions for the existence of at least three solutions to the above problem by using the Avery and Peterson fixed point theorem.

However, there are not many concerning the boundary value problems, in which a generalized $p$-Laplacian equation with a nonlocal boundary conditions. The motivation for the present work stems from many recent investigations in $[16,23-25]$ and references therein. Our purpose of this paper is to establish some sufficient conditions for the existence of 
triple positive solutions to a class of a singular generalized one-dimensional $p$-Laplacian problem

$$
\begin{gathered}
\left(r(t) \varphi_{p}\left(u^{\prime}\right)\right)^{\prime}+h(t) f\left(t, u(t), u^{\prime}(t)\right)=0, \quad t \in(a, b), \\
\alpha_{1} u(a)-\beta_{1} u^{\prime}(a)=\sum_{i=1}^{m-2} \gamma_{i} u\left(\xi_{i}\right), \quad u^{\prime}(b)=0
\end{gathered}
$$

where $\varphi_{p}(u)$ is $p$-Laplacian operator; that is, $\varphi_{p}(u)=|u|^{p-2} u$, $p>1,\left(\varphi_{p}\right)^{-1}(u)=\varphi_{q}(u), 1 / p+1 / q=1$, and $\alpha_{1}, \beta_{1}, \gamma_{i}$, $\xi_{i}, r, h, f$ satisfy

$\left(A_{1}\right) \alpha_{1}, \beta_{1} \in[0, \infty), \gamma_{i} \in[0, \infty)$ satisfy $\alpha_{1}>\sum_{i=1}^{m-2} \gamma_{i}, a<$ $\xi_{1}<\cdots<\xi_{m-2}<b(m>3)$,

$\left(A_{2}\right) r(t) \in C^{1}(a, b), r(t)$ is positive and nondecreasing on $[a, b]$

$\left(A_{3}\right) h \in L^{1}((a, b),[0, \infty))$ may be singular at $t=0$ and/or $t=1$, and $0<\int_{\Gamma} h(t) d t<\infty$, where $\Gamma \subseteq[a, b]$,

$\left(A_{4}\right) f:[a, b] \times[0, \infty) \times(-\infty, \infty) \rightarrow[0, \infty)$ is an $L^{1}$-Carathédory function; that is, for each $(t, x, y) \in$ $[a, b] \times[0, \infty) \times(-\infty, \infty)$, the mapping $t \rightarrow f(t, x, y)$ is Lebesgue measurable on $[a, b]$; for a.e. $t \in[a, b]$, the mapping $(t, x, y) \rightarrow f(t, x, y)$ is continuous on $[0, \infty) \times(-\infty, \infty)$.

Under the above assumptions, some new and general results on the existence of multiple positive solutions to singular one-dimensional $p$-Laplacian are obtained. Our results develop some results of Cheung and Ren [23] and include and improve the main results of Feng and Ge [16] and Zhang [25].

The rest of the paper is organized as follows. In Section 2, we provide some background material from the theory of cones in the Banach spaces, which are useful later. Some lemmas and criteria for the existence of three positive solutions for one-dimensional $p$-Laplacian problems are established in Section 3. Finally, we give an example to illustrate our main results.

\section{Preliminaries}

Let $\gamma$ and $\theta$ be nonnegative continuous convex functionals on a cone $K, \alpha$ a nonnegative continuous concave functional on $K, \beta$ a nonnegative continuous functional on $K$, and $m_{1}, m_{2}, m_{3}$, and $m_{4}$ positive numbers. We define the following convex sets and a closed set

$$
\begin{gathered}
P\left(\gamma, m_{4}\right)=\left\{u \in K: \gamma(u)<m_{4}\right\}, \\
P\left(\gamma, \alpha, m_{2}, m_{4}\right)=\left\{u \in K: m_{2} \leq \alpha(u), \gamma(u) \leq m_{4}\right\}, \\
P\left(\gamma, \theta, \alpha, m_{2}, m_{3}, m_{4}\right) \\
=\left\{u \in K: m_{2} \leq \alpha(u), \theta(u) \leq m_{3}, \gamma(u) \leq m_{4}\right\}, \\
Q\left(\gamma, \beta, m_{1}, m_{4}\right)=\left\{u \in K: m_{1} \leq \beta(u), \gamma(u) \leq m_{4}\right\} .
\end{gathered}
$$

Now we state the fixed point theorem due to Avery and Peterson [26].
Lemma 1. Let $K$ be a cone in a real Banach space E. Let $\gamma$ and $\theta$ be nonnegative continuous convex functionals on $K, \alpha$ a nonnegative continuous concave functional on $K$, and $\beta$ a nonnegative continuous functional on $K$ satisfying $\beta(\rho u) \leq$ $\rho \beta(u)$ for $0 \leq \rho \leq 1$, such that, for some positive numbers $\epsilon$ and $m_{4}$,

$$
\alpha(u) \leq \beta(u), \quad\|u\| \leq \epsilon \gamma(u) \quad \forall u \in \overline{P\left(\gamma, m_{4}\right)}
$$

Suppose that $T: \overline{P\left(\gamma, m_{4}\right)} \rightarrow \overline{P\left(\gamma, m_{4}\right)}$ is completely continuous and there are positive numbers $m_{1}, m_{2}$, and $m_{3}$ with $m_{1}<$ $m_{2}$ such that

$\left(B_{1}\right)\left\{u \in P\left(\gamma, \theta, \alpha, m_{2}, m_{3}, m_{4}\right): \alpha(u)>m_{2}\right\} \neq \emptyset, \alpha(T u)>$ $m_{2}$ for $u \in P\left(\gamma, \theta, \alpha, m_{2}, m_{3}, m_{4}\right)$,

$\left(B_{2}\right) \alpha(T u)>m_{2}$ for $u \in P\left(\gamma, \alpha, m_{2}, m_{4}\right)$ with $\theta(T u)>m_{3}$,

$\left(B_{3}\right) 0 \notin Q\left(\gamma, \beta, m_{1}, m_{4}\right)$ and $\beta(T u)<m_{1}$ for $u \in Q(\gamma, \beta$, $\left.m_{1}, m_{4}\right)$ with $\beta(u)=m_{1}$.

Then $T$ has at least three fixed points $u_{1}, u_{2}, u_{3} \in \overline{P\left(\gamma, m_{4}\right)}$ such that

$$
\begin{gathered}
\gamma\left(u_{i}\right) \leq m_{4} \quad(i=1,2,3) \\
m_{2}<\alpha\left(u_{1}\right), \quad m_{1}<\beta\left(u_{2}\right) \quad \text { with } \alpha\left(u_{2}\right)<m_{2}, \\
\beta\left(u_{3}\right)<m_{1} .
\end{gathered}
$$

Denote that $L^{+}[a, b]=\{v \in L[a, b]: v(t) \geq 0, t \in[a, b]\}$.

Lemma 2. Assume that $\left(A_{1}\right)$ and $\left(A_{2}\right)$ hold. If $g \in L^{+}[a, b]$, then the boundary value problem

$$
\begin{gathered}
\left(r(t) \varphi_{p}\left(u^{\prime}\right)\right)^{\prime}+g(t)=0, \quad t \in(a, b), \\
\alpha_{1} u(a)-\beta_{1} u^{\prime}(a)=\sum_{i=1}^{m-2} \gamma_{i} u\left(\xi_{i}\right), \quad u^{\prime}(b)=0,
\end{gathered}
$$

has a unique solution

$$
\begin{aligned}
u(t)= & \int_{a}^{t} \varphi_{q}\left(\frac{1}{r(s)} \int_{s}^{b} g(\tau) d \tau\right) d s+\frac{1}{\alpha_{1}-\sum_{i=1}^{m-2} \gamma_{i}} \\
& \times\left(\beta_{1} \varphi_{q}\left(\frac{1}{r(a)} \int_{a}^{b} g(s) d s\right)\right. \\
& \left.+\sum_{i=1}^{m-2} \gamma_{i} \int_{a}^{\xi_{i}} \varphi_{q}\left(\frac{1}{r(s)} \int_{s}^{b} g(\tau) d \tau\right) d s\right) .
\end{aligned}
$$

Proof. For a.e. $t \in[a, b]$, integrating (7) from $t$ to $b$, in view of (8), we have

$$
u^{\prime}(t)=\varphi_{q}\left(\frac{1}{r(t)} \int_{t}^{b} g(s) d s\right) .
$$

Integrating this from $a$ to $t$ yields

$$
u(t)=u(a)+\int_{a}^{t} \varphi_{q}\left(\frac{1}{r(s)} \int_{s}^{b} g(\tau) d \tau\right) d s
$$


Again, it follows from (8) that

$$
\begin{aligned}
u(a)= & \frac{1}{\alpha_{1}-\sum_{i=1}^{m-2} \gamma_{i}} \\
& \times\left(\beta_{1} \varphi_{q}\left(\frac{1}{r(a)} \int_{a}^{b} g(s) d s\right)\right. \\
& \left.\quad+\sum_{i=1}^{m-2} \gamma_{i} \int_{a}^{\xi_{i}} \varphi_{q}\left(\frac{1}{r(s)} \int_{s}^{b} g(\tau) d \tau\right) d s\right) .
\end{aligned}
$$

The proof is complete.

Lemma 3. Assume that $\left(A_{1}\right)$ and $\left(A_{2}\right)$ hold. If $g \in L^{+}[a, b]$, then the unique solution of the problems (7) and (8) satisfies

$$
u(t) \geq 0, \quad \min _{t \in[\sigma, b-\sigma]} u(t) \geq \tau_{1} \max _{t \in[a, b]} u(t),
$$

where $\tau_{1}=\beta_{1} /\left(\beta_{1}+(b-a)\left(\alpha_{1}-\sum_{i=1}^{m-2} \gamma_{i}\right)\right), \sigma \in(a,(a+b) / 2)$.

Proof. The proof is similar to Lemma 3.6 in [25], we omit it.

Let $E$ be the Banach space $C^{1}[a, b]$ with the norm $\|u\|=$ $\max \left\{\|u\|_{0},\left\|u^{\prime}\right\|_{0}\right\}$, where $\|u\|_{0}=\max _{0 \leq t \leq 1}|u(t)|$. Define a cone $K \subset E$ by

$$
\begin{aligned}
& K=\{u \in E: u(t) \geq 0 \text { for } t \in[a, b], \\
& u^{\prime \prime}(t) \leq 0, u^{\prime}(t) \geq 0 \text { for } t \in(a, b), \\
& \alpha_{1} u(a)-\beta_{1} u^{\prime}(a)=\sum_{i=1}^{m-2} \gamma_{i} u\left(\xi_{i}\right), \\
& \left.u^{\prime}(b)=0, \min _{t \in[\sigma, b-\sigma]} u(t) \geq \tau_{1} \max _{t \in[a, b]} u(t)\right\} .
\end{aligned}
$$

Let the assumptions $\left(A_{1}\right)$ and $\left(A_{2}\right)$ hold. Denote that

$$
\begin{aligned}
\lambda= & \int_{a}^{b}\left(\frac{b-s}{r(s)}\right)^{1 /(p-1)} d s+\frac{1}{\alpha_{1}-\sum_{i=1}^{m-2} \gamma_{i}} \\
& \times\left(\beta_{1}\left(\frac{b-a}{r(a)}\right)^{1 /(p-1)}+\sum_{i=1}^{m-2} \gamma_{i} \int_{a}^{\xi_{i}}\left(\frac{b-s}{r(s)}\right)^{1 /(p-1)} d s\right) .
\end{aligned}
$$

Evidently, $\lambda \geq 0$. We take

$$
\begin{aligned}
u_{0}(t)= & \int_{a}^{t}\left(\bar{m} \frac{b-s}{r(s)}\right)^{1 /(p-1)} d s+\frac{1}{\alpha_{1}-\sum_{i=1}^{m-2} \gamma_{i}} \\
& \times\left(\beta_{1}\left(\bar{m} \frac{b-a}{r(a)}\right)^{1 /(p-1)}\right. \\
& \left.+\sum_{i=1}^{m-2} \gamma_{i} \int_{a}^{\xi_{i}}\left(\bar{m} \frac{b-s}{r(s)}\right)^{1 /(p-1)} d s\right),
\end{aligned}
$$

where $\bar{m}$ satisfies $(\bar{m})^{1 /(p-1)}:=m_{2} / \tau_{1}^{2} \lambda, m_{2}>0$ is constant. It is not difficult to check that $u_{0}(t) \geq 0$ for a.e. $t \in[a, b]$, $u^{\prime \prime}(t) \leq 0, u^{\prime}(t) \geq 0$ for a.e. $t \in(a, b)$, and $u_{0}(t)$ satisfies $\alpha_{1} u_{0}(a)-\beta_{1} u_{0}^{\prime}(a)=\sum_{i=1}^{m-2} \gamma_{i} u_{0}\left(\xi_{i}\right), u_{0}^{\prime}(b)=0$. That is to say $u_{0} \in K$. Hence, $K \backslash\{0\} \neq \emptyset$.

To obtain the existence of solutions for the problem $(\mathscr{P})$, the following priori estimate is needful.

Lemma 4. Assume that $\left(A_{1}\right)$ holds. If $u \in K$, then

$$
\max _{t \in[a, b]} u(t) \leq \tau_{2} \max _{t \in[a, b]}\left|u^{\prime}(t)\right|
$$

where $\tau_{2}=\left(\beta_{1}+\alpha_{1}(b-a)\right) /\left(\alpha_{1}-\sum_{i=1}^{m-2} \gamma_{i}\right)$.

Proof. The proof is similar to Lemma 3.1 in [21], we omit it.

Let the nonnegative continuous convex functionals $\gamma$ and $\theta$, the nonnegative continuous concave functional $\alpha$, and the nonnegative continuous functional $\beta$ be defined on cone $K$ by

$$
\begin{gathered}
\alpha(u)=\min _{t \in[\sigma, b-\sigma]} u(t), \quad \beta(u)=\theta(u)=\max _{t \in[a, b]} u(t), \\
\gamma(u)=\max _{t \in[a, b]}\left|u^{\prime}(t)\right| .
\end{gathered}
$$

In the view of assumption $\left(A_{3}\right)$, that is, $0<\int_{a}^{b} h(\tau) d \tau<$ $\infty$ and $\left(A_{3}\right)$. Thus, we can define an operator $T$ by

$(T u)(t)$

$$
\begin{aligned}
& :=\int_{a}^{t} \varphi_{q}\left(\frac{1}{r(s)} \int_{s}^{b} h(\tau) f\left(\tau, u(\tau), u^{\prime}(\tau)\right) d \tau\right) d s \\
& +\frac{1}{\alpha_{1}-\sum_{i=1}^{m-2} \gamma_{i}} \\
& \quad \times\left(\beta_{1} \varphi_{q}\left(\frac{1}{r(a)} \int_{a}^{b} h(\tau) f\left(\tau, u(\tau), u^{\prime}(\tau)\right) d \tau\right)\right. \\
& \quad+\sum_{i=1}^{m-2} \gamma_{i} \int_{a}^{\xi_{i}} \varphi_{q}\left(\frac{1}{r(s)} \int_{s}^{b} h(\tau)\right. \\
& \left.\left.\quad \times f\left(\tau, u(\tau), u^{\prime}(\tau)\right) d \tau\right) d s\right)
\end{aligned}
$$

for a.e. $t \in[a, b]$.

Lemma 5. Assume that $\left(A_{1}\right)-\left(A_{4}\right)$ hold. Then $T: K \rightarrow K$ is completely continuous.

Proof. For any $u \in K$, by the definition of $T u$, we see that $T u \in C^{1}[a, b],\left(\varphi_{p}\left((T u)^{\prime}\right)\right)^{\prime} \in L^{1}[a, b]$. From $\left(A_{1}\right)-\left(A_{4}\right)$ and Lemma 3, we have $(T u)(t) \geq 0,\left(\varphi_{p}\left((T u)^{\prime}\right)\right)^{\prime} \leq 0$ implies that $(T u)(t)$ is concave on $[a, b],-\left(\varphi_{p}\left((T u)^{\prime}\right)\right)^{\prime}=h(t) f(t,(T u)(t)$, $\left.(T u)^{\prime}(t)\right) \geq 0, \alpha_{1} T u(a)-\beta_{1} T u^{\prime}(a)=\sum_{i=1}^{m-2} \gamma_{i} T u\left(\xi_{i}\right)$, and 
$T u^{\prime}(b)=0$. So we can conclude that $T u \in K$. In what follows, we prove that $T$ is compact. Let $R>0$, there exists a set $\Omega$ such that $\Omega \subset\left\{u \in K:\left\|u^{\prime}\right\|_{0} \leq R\right\}$. For any $u \in \Omega$, the assumptions imply that

$$
\begin{aligned}
0 & \leq \int_{a}^{b} h(t) f\left(t, u(t), u^{\prime}(t)\right) d t \\
& \leq \max _{\left(t, u, u^{\prime}\right) \in[a, b] \times\left[0, \tau_{2} R\right] \times[-R, R]} f\left(t, u, u^{\prime}\right) \int_{a}^{b} h(t) d t<\infty .
\end{aligned}
$$

We take the arguments to show that the operator $T$ is completely continuous. In view of Lemma 4 and the continuity of $f$ in $u$ and $u^{\prime}$, assume that $u_{n}, u_{0} \in K$ satisfy $\| u_{n}$ $u_{0} \| \rightarrow 0(n \rightarrow \infty)$. Moreover, $\varphi_{q}(v)$ is an increasing and continuously function, and we obtain that

$$
\begin{aligned}
& \left\|T u_{n}-T u_{0}\right\|_{0} \\
& =\max _{a \leq t \leq b}\left|T u_{n}(t)-T u_{0}(t)\right| \\
& \leq \int_{a}^{b}\left(\varphi_{q}\left(\frac{1}{r(s)} \int_{s}^{b} h(\tau) f\left(\tau, u_{n}(\tau), u_{n}^{\prime}(\tau)\right) d \tau\right)\right. \\
& \left.\left.+\frac{\beta_{1}}{\alpha_{1}-\sum_{i=1}^{m-2} \gamma_{i}} \frac{1}{r(s)} \int_{s}^{b} h(\tau) f\left(\tau, u_{0}(\tau), u_{0}^{\prime}(\tau)\right) d \tau\right)\right) d s \\
& \times\left(\varphi_{q}\left(\frac{1}{r(a)} \int_{a}^{b} h(\tau) f\left(\tau, u_{n}(\tau), u_{n}^{\prime}(\tau)\right) d \tau\right)\right. \\
& \left.\quad-\varphi_{q}\left(\frac{1}{r(a)} \int_{a}^{b} h(\tau) f\left(\tau, u_{0}(\tau), u_{0}^{\prime}(\tau)\right) d \tau\right)\right) \\
& +\frac{1}{\alpha_{1}-\sum_{i=1}^{m-2} \gamma_{i}} \sum_{i=1}^{m-2} \gamma_{i} \\
& \times \int_{a}^{\xi_{i}}\left(\varphi_{q}\left(\frac{1}{r(s)} \int_{s}^{b} h(\tau) f\left(\tau, u_{n}(\tau), u_{n}^{\prime}(\tau)\right) d \tau\right) d s\right. \\
& \quad-\varphi_{q}\left(\frac{1}{r(s)} \int_{s}^{b} h(\tau)\right.
\end{aligned}
$$

$$
\begin{aligned}
& \left\|\left(T u_{n}\right)^{\prime}-\left(T u_{0}\right)^{\prime}\right\|_{0} \\
& =\max _{a \leq t \leq b}\left|\left(T u_{n}\right)^{\prime}(t)-\left(T u_{0}\right)^{\prime}(t)\right| \\
& \leq \mid \varphi_{q}\left(\frac{1}{r(a)} \int_{a}^{b} h(\tau) f\left(\tau, u_{n}(\tau), u_{n}^{\prime}(\tau)\right) d \tau\right) \\
& \quad-\varphi_{q}\left(\frac{1}{r(a)} \int_{a}^{b} h(\tau) f\left(\tau, u_{0}(\tau), u_{0}^{\prime}(\tau)\right) d \tau\right) \mid \rightarrow 0 \\
& \quad(n \rightarrow \infty) .
\end{aligned}
$$

Therefore,

$$
\left\|T u_{n}-T u_{0}\right\| \longrightarrow 0 \quad(n \longrightarrow \infty) .
$$

This means that the operator $T: K \rightarrow K$ is continuous.

Since $h(t)$ may be singular at $t=a$ and/or $t=b$, choose two sequences $\left\{\mu_{n}\right\}_{n=1}^{\infty},\left\{v_{n}\right\}_{n=1}^{\infty} \subset(a, b)$ satisfying $\mu_{n} \leq v_{n}$ for any $n \geq 1$, such that $\mu_{n} \rightarrow a$ and $\alpha_{n} \rightarrow b$ as $n \rightarrow \infty$, respectively. Define

$$
h_{n}(t)= \begin{cases}\inf _{a \leq t \leq \mu_{n}} h(t), & a \leq t \leq \mu_{n}, \\ h(t), & \mu_{n} \leq t \leq v_{n}, \\ \inf _{v_{n} \leq t \leq b} h(t), & v_{n} \leq t \leq b,\end{cases}
$$

and an operator sequence $T_{n}$ by

$$
\begin{aligned}
& \left(T_{n} u\right)(t) \\
& =\int_{a}^{t} \varphi_{q}\left(\frac{1}{r(s)} \int_{s}^{b} h_{n}(\tau) f\left(\tau, u(\tau), u^{\prime}(\tau)\right) d \tau\right) d s \\
& +\frac{1}{\alpha_{1}-\sum_{i=1}^{m-2} \gamma_{i}} \\
& \quad \times\left(\beta_{1} \varphi_{q}\left(\frac{1}{r(a)} \int_{a}^{b} h_{n}(\tau) f\left(\tau, u(\tau), u^{\prime}(\tau)\right) d \tau\right)\right. \\
& \quad+\sum_{i=1}^{m-2} \gamma_{i} \\
& \quad \times \int_{a}^{\xi_{i}} \varphi_{q}\left(\frac{1}{r(s)} \int_{s}^{b} h_{n}(\tau)\right. \\
& \left.\left.\times f\left(\tau, u(\tau), u^{\prime}(\tau)\right) d \tau\right) d s\right) \\
& \quad \text { for a.e. } t \in[a, b] .
\end{aligned}
$$

Clearly, $h_{n}(t):[a, b] \rightarrow[0, \infty)$ is a piecewise continuous function, and the operator $T_{n}: K \rightarrow K$ is well defined. Further, we can see that $T_{n}: K \rightarrow K$ is completely continuous. 
Let $r>0, \mathbb{B}_{r}:=\left\{u \in K:\left\|u^{\prime}\right\|_{0} \leq r\right\}$ and $M_{r}=$ $\max \left\{f\left(t, u, u^{\prime}\right): u \in K, t \in[a, b], u \in\left[0, \tau_{2} r\right], u^{\prime} \in[-r, r]\right\}$. We will prove that $T_{n}$ approaches $T$ uniformly on $\mathbb{B}_{r}$. From the absolute continuity of integral, we obtain

$$
\lim _{n \rightarrow \infty} \int_{\Gamma(n)} \varphi_{q}\left(\frac{1}{r(s)} \int_{s}^{b} h(\tau) f\left(\tau, u(\tau), u^{\prime}(\tau)\right) d \tau\right) d s=0,
$$

where $\Gamma(n)=\left[a, \mu_{n}\right] \cup\left[v_{n}, b\right]$. For any $u \in \mathbb{B}_{r}$ and a.e. $t \in$ $\left[a, \mu_{n}\right]$, one has that

$$
\begin{aligned}
& \left\|T_{n} u-T u\right\|_{0} \\
& =\max _{a \leq t \leq \mu_{n}}\left|T_{n} u(t)-T u(t)\right| \\
& \leq \int_{a}^{\mu_{n}} \varphi_{q}\left(\frac{1}{r(s)} \int_{s}^{b} h_{n}(\tau) f\left(\tau, u(\tau), u^{\prime}(\tau)\right) d \tau\right) d s \\
& \quad-\varphi_{q}\left(\frac{1}{r(s)} \int_{s}^{b} h(\tau) f\left(\tau, u(\tau), u^{\prime}(\tau)\right) d \tau\right) d s \\
& +\frac{\beta_{1}}{\alpha_{1}-\sum_{i=1}^{m-2} \gamma_{i}} \\
& \quad \times\left(\varphi_{q}\left(\frac{1}{r(a)} \int_{a}^{b} h_{n}(\tau) f\left(\tau, u(\tau), u^{\prime}(\tau)\right) d \tau\right)\right. \\
& \quad+\int_{\Gamma(n)} \varphi_{q}\left(\frac{1}{r(s)} \int_{s}^{b} h_{n}(\tau) f\left(\tau, u(\tau), u^{\prime}(\tau)\right) d \tau\right) d s \\
& \quad-\varphi_{q}\left(\frac{1}{r(s)} \int_{s}^{b} h(\tau) f\left(\tau, u(\tau), u^{\prime}(\tau)\right) d \tau\right) d s \\
& \left.\quad+\frac{1}{\alpha_{1}-\sum_{i=1}^{m-2} \gamma_{i}} \sum_{i=1}^{m-2} \gamma_{i} h_{n}(\tau) f\left(\tau, u(\tau), u^{\prime}(\tau)\right) d \tau\right) \\
& \quad \times \int_{a}^{\xi_{i}}\left(\varphi_{q}\left(\frac{1}{r(s)} \int_{a}^{b} h(\tau) f\left(\tau, u(\tau), u^{\prime}(\tau)\right) d \tau\right)\right) \\
& \left.\quad-\varphi_{q}\left(\frac{1}{r(s)} \int_{s}^{b} h(\tau) f\left(\tau, u(\tau), u^{\prime}(\tau)\right) d \tau\right) d s\right)
\end{aligned}
$$

$$
\begin{gathered}
\left.-\varphi_{q}\left(\frac{1}{r(a)} \int_{a}^{b} h(\tau) f\left(\tau, u(\tau), u^{\prime}(\tau)\right) d \tau\right)\right) \\
+\frac{1}{\alpha_{1}-\sum_{i=1}^{m-2} \gamma_{i}} \sum_{i=1}^{m-2} \gamma_{i} \\
\times \int_{a}^{\xi_{i}}\left(\varphi_{q}\left(\frac{1}{r(s)} \int_{s}^{b} h_{n}(\tau) f\left(\tau, u(\tau), u^{\prime}(\tau)\right) d \tau\right) d s\right. \\
\left.-\varphi_{q}\left(\frac{1}{r(s)} \int_{s}^{b} h(\tau) f\left(\tau, u(\tau), u^{\prime}(\tau)\right) d \tau\right) d s\right) .
\end{gathered}
$$

For $u \in \mathbb{B}_{r}$ and a.e. $t \in\left[v_{n}, b\right]$, we have the same result. It is easy to see that, for any $u \in \mathbb{B}_{r}$ and a.e. $t \in\left[\mu_{n}, v_{n}\right]$, there is $\left\|T_{n} u-T u\right\|_{0}=0$. Similarly, we can obtain that, for any $u \in \mathbb{B}_{r}$ and a.e. $t \in\left[a, \mu_{n}\right], t \in\left[\mu_{n}, v_{n}\right], t \in\left[v_{n}, b\right]$, respectively,

$$
\begin{aligned}
& \left\|\left(T_{n} u\right)^{\prime}-(T u)^{\prime}\right\|_{0} \\
& =\max _{t}\left|\left(T_{n} u\right)^{\prime}(t)-(T u)^{\prime}(t)\right| \\
& \leq \mid \varphi_{q}\left(\frac{1}{r(a)} \int_{a}^{b} h_{n}(\tau) f\left(\tau, u(\tau), u^{\prime}(\tau)\right) d \tau\right) \\
& \quad-\varphi_{q}\left(\frac{1}{r(a)} \int_{a}^{b} h(\tau) f\left(\tau, u(\tau), u^{\prime}(\tau)\right) d \tau\right) \mid .
\end{aligned}
$$

In view of the continuity of function $\varphi_{q}$, from the above arguments, we have

$$
\left\|T_{n} u-T u\right\| \longrightarrow 0 \quad(n \longrightarrow \infty) .
$$

That is to say, the sequence $T_{n}$ is uniformly an approximate $T$ on any bounded subset of $K$. Applying the Arzela-Ascoli lemma to the operator $T$, we can conclude that $T \Omega$ is relatively compact; that is, $T$ is completely continuous.

\section{Main Results}

We are ready to apply the Avery and Peterson fixed point theorem to the operator $T$ to give sufficient conditions for the existence of at least three positive solutions to the problem $(\mathscr{P})$. For convenience, we introduce the following notation:

$$
\begin{gathered}
L=\frac{1}{r(a)} \int_{a}^{b} h(\tau) d \tau, \quad M=\frac{\alpha_{1}-\sum_{i=1}^{m-2} \gamma_{i}}{\beta_{1}}, \\
N=b-a+\frac{\beta_{1}+\sum_{i=1}^{m-2} \gamma_{i}\left(\xi_{i}-a\right)}{\alpha_{1}-\sum_{i=1}^{m-2} \gamma_{i}} .
\end{gathered}
$$

Theorem 6. Suppose $\left(A_{1}\right)-\left(A_{4}\right)$ hold and $f(t, 0,0) \not \equiv$ for a.e. $t \in[a, b]$. If there exist positive numbers $m_{1}, m_{2}$, and $m_{4}$ with $m_{1}<m_{2} \leq \tau_{1}^{2} \lambda((b-a) / r(a))^{-1 /(p-1)} m_{4}$ such that the following conditions are satisfied:

$\left(C_{1}\right) f(t, \mu, \nu) \leq(1 / L) \varphi_{p}\left(m_{4}\right),(t, \mu, \nu) \in[a, b] \times\left[0, \tau_{2} m_{4}\right] \times$ $\left[-m_{4}, m_{4}\right]$, 
$\left(C_{2}\right) f(t, \mu, v)>(1 / L) \varphi_{p}\left(m_{2} / \tau_{1} M\right),(t, \mu, \nu) \in[\sigma, b-\sigma] \times$ $\left[m_{2}, m_{2} \tau_{1}^{-2}\right] \times\left[-m_{4}, m_{4}\right]$

$\left(C_{3}\right) f(t, \mu, v) \leq(1 / L) \varphi_{p}\left(m_{1} / N\right),(t, \mu, \nu) \in[a, b] \times$ $\left[0, m_{1}\right] \times\left[-m_{4}, m_{4}\right]$

then the problem $(\mathscr{P})$ has at least three positive solutions $u_{1}$, $u_{2}$, and $u_{3}$ satisfying

$$
\begin{gathered}
\max _{t \in[a, b]}\left|u_{i}^{\prime}(t)\right| \leq m_{4} \quad \text { for } i=1,2,3, \\
m_{2}<\min _{t \in[\sigma, b-\sigma]} u_{1}(t), \quad m_{1}<\max _{t \in[a, b]} u_{2}(t) \\
\text { with } \min _{t \in[\sigma, b-\sigma]} u_{2}(t)<m_{2}, \\
\max _{t \in[a, b]} u_{3}(t)<m_{1} .
\end{gathered}
$$

Proof. It is known that boundary value problem $(\mathscr{P})$ has a solution $u$ if and only if $u$ solves the operator equation $u=T u$. By the definition of operator $T$, from Lemma 4 and the concavity of $u$, the functionals defined above satisfy $\sigma(b-\sigma) \theta(u) \leq \alpha(u) \leq \theta(u)=\beta(u),\|u\|=\max \{\theta(u), \gamma(u)\} \leq$ $\max \left\{1, \tau_{2}\right\} \gamma(u)$ for any $u \in \overline{P\left(\gamma, m_{4}\right)} \subset K$. We also note that $\beta(\rho u) \leq \rho \beta(u)$ for $0 \leq \rho \leq 1$, which suffices to show that the conditions of Lemma 1 hold with respect to $T$. First of all, we show that if the assumption $\left(C_{1}\right)$ is satisfied, then

$$
T: \overline{P\left(\gamma, m_{4}\right)} \longrightarrow \overline{P\left(\gamma, m_{4}\right)} .
$$

In fact, for $u \in \overline{P\left(\gamma, m_{4}\right)}$, there is $\gamma(u)=\max _{t \in[a, b]}\left|u^{\prime}(t)\right| \leq$ $m_{4}$. With Lemma 4, there is $\max _{t \in[a, b]} u(t) \leq$ $\tau_{2} \max _{t \in[a, b]}\left|u^{\prime}(t)\right| \leq \tau_{2} m_{4}$, and the assumption $\left(C_{1}\right)$ implies that $f\left(t, u(t), u^{\prime}(t)\right) \leq(1 / L) \varphi_{p}\left(m_{4}\right)$ for a.e. $t \in[a, b]$. On the other hand, for $u \in K$, we obtain that $T u \in K$, and so $(T u)(t)$ is concave on $[a, b]$, and there is $\max _{t \in[a, b]}\left|(T u)^{\prime}(t)\right|=\left|(T u)^{\prime}(a)\right|$. By using of $r(t)$ is positive and monotone increasing on a.e. $[a, b]$, and so

$$
\begin{aligned}
\gamma(T u) & =\max _{t \in[a, b]}\left|(T u)^{\prime}(t)\right| \\
& =\varphi_{q}\left(\frac{1}{r(a)} \int_{a}^{b} h(\tau) f\left(\tau, u(\tau), u^{\prime}(\tau)\right) d \tau\right) \\
& \leq \varphi_{q}\left(\frac{1}{L} \varphi_{p}\left(m_{4}\right) \frac{1}{r(a)} \int_{a}^{b} h(\tau) d \tau\right)=m_{4} .
\end{aligned}
$$

Therefore, (31) is satisfied.

To check if the condition $\left(B_{1}\right)$ in Lemma 1 is fulfilled, we choose $u_{0}(t)$, where $u_{0}(t)$ is defined by (16). From the hypothesis conditions of the theorem, we have

$$
\begin{gathered}
\alpha\left(u_{0}\right)=\min _{t \in[\sigma, b-\sigma]} u_{0}(t)=u_{0}(\sigma) \geq \tau_{1}(\bar{m})^{1 /(p-1)} \lambda \\
\geq m_{2} \tau_{1}^{-1}>m_{2}, \\
\theta\left(u_{0}\right)=\max _{t \in[a, b]} u_{0}(t)=u_{0}(b)=(\bar{m})^{1 /(p-1)} \lambda>m_{2} \tau_{1}^{-2}>m_{2},
\end{gathered}
$$

$$
\begin{aligned}
\gamma\left(u_{0}\right) & =\max _{t \in[a, b]}\left|\left(u_{0}\right)^{\prime}(t)\right|=\left|\left(u_{0}\right)^{\prime}(a)\right| \\
& =\left(\bar{m} \frac{b-a}{r(a)}\right)^{1 /(p-1)}=\frac{m_{2} \tau_{1}^{-2}}{\lambda}\left(\frac{b-a}{r(a)}\right)^{1 /(p-1)} \leq m_{4} .
\end{aligned}
$$

Equation (33) implies that $\alpha\left(u_{0}\right) \geq \tau_{1} \theta\left(u_{0}\right)$. That is, $\min _{t \in[\sigma, b-\sigma]} u_{0}(t) \geq \tau_{1} \max _{t \in[a, b]} u_{0}(t)$. So for $u_{0} \in K, u_{0} \in$ $P\left(\gamma, \theta, \alpha, m_{2}, m_{2} \tau_{1}^{-2}, m_{4}\right)$ and $\left\{u_{0} \in P\left(\gamma, \theta, \alpha, m_{2}, m_{2} \tau_{1}^{-2}, m_{4}\right):\right.$ $\left.\alpha\left(u_{0}\right)>m_{2}\right\} \neq \emptyset$ hold. Hence, for $u \in P\left(\gamma, \theta, \alpha, m_{2}, m_{2} \tau_{1}^{-2}\right.$, $\left.m_{4}\right)$, we see that $m_{2} \leq u(t) \leq m_{2} \tau_{1}^{-2}$ and $\left|u^{\prime}(t)\right| \leq m_{4}$ for a.e. $t \in[\sigma, b-\sigma]$. Hence by the assumption $\left(C_{2}\right)$, one has that $f\left(t, u(t), u^{\prime}(t)\right)>(1 / L) \varphi_{p}\left(m_{2} \tau_{1}^{-1} / M\right)$ for a.e. $t \in[\sigma, b-\sigma]$. By the definition of the functional $\alpha$, we obtain

$$
\begin{aligned}
& \alpha(T u)=\min _{t \in[\sigma, b-\sigma]}(T u)(t) \geq \tau_{1} \max _{t \in[a, b]}(T u)(t)=\tau_{1}(T u)(b) \\
& =\tau_{1}\left(\int_{a}^{b} \varphi_{q}\left(\frac{1}{r(s)} \int_{s}^{b} h(\tau) f\left(\tau, u(\tau), u^{\prime}(\tau)\right) d \tau\right) d s\right. \\
& +\frac{\beta_{1}}{\alpha_{1}-\sum_{i=1}^{m-2} \gamma_{i}} \varphi_{q} \\
& \times\left(\frac{1}{r(a)} \int_{a}^{b} h(\tau) f\left(\tau, u(\tau), u^{\prime}(\tau)\right) d \tau\right) \\
& +\frac{1}{\alpha_{1}-\sum_{i=1}^{m-2} \gamma_{i}} \sum_{i=1}^{m-2} \gamma_{i} \\
& \times \int_{a}^{\xi_{i}} \varphi_{q}\left(\frac{1}{r(s)} \int_{s}^{b} h(\tau)\right. \\
& \left.\left.\times f\left(\tau, u(\tau), u^{\prime}(\tau)\right) d \tau\right) d s\right) \\
& \geq \frac{\tau_{1} \beta_{1}}{\alpha_{1}-\sum_{i=1}^{m-2} \gamma_{i}} \varphi_{q}\left(\frac{1}{L} \varphi_{p}\left(\frac{m_{2} \tau_{1}^{-1}}{M}\right) \frac{1}{r(a)}\right. \\
& \left.\times \int_{a}^{b} h(\tau) d \tau\right)=m_{2}
\end{aligned}
$$

Therefore, we get $\alpha(T u)>m_{2}$ for $u \in P\left(\gamma, \theta, \alpha, m_{2}, m_{2} \tau_{1}^{-2}\right.$, $\left.m_{4}\right)$.

Next, we show that the condition $\left(B_{2}\right)$ in Lemma 1 holds. In fact, if $u \in P\left(\gamma, \alpha, m_{2}, m_{4}\right)$ with $\theta(T u)>m_{2} \tau_{1}^{-2}$, then

$$
\begin{aligned}
\alpha(T u) & =\min _{t \in[\sigma, b-\sigma]}(T u)(t) \geq \tau_{1} \max _{t \in[a, b]}(T u)(t) \\
& =\tau_{1} \theta(T u)>m_{2} \tau_{1}^{-1}>m_{2} .
\end{aligned}
$$

Finally, we assert that the condition $\left(B_{3}\right)$ in Lemma 1 also holds. Since $\beta(0)=0<m_{1}$, there holds that $0 \notin Q(\gamma, \beta$, 
$\left.m_{1}, m_{4}\right)$. Assume that $u \in Q\left(\gamma, \beta, m_{1}, m_{4}\right)$ with $\beta(u)=m_{1}$. Then, by the assumption $\left(C_{3}\right)$ we get

$$
\begin{aligned}
& \beta(T u)=\max _{t \in[a, b]}(T u)(t)=(T u)(b) \\
& =\int_{a}^{b} \varphi_{q}\left(\frac{1}{r(s)} \int_{s}^{b} h(\tau) f\left(\tau, u(\tau), u^{\prime}(\tau)\right) d \tau\right) d s \\
& +\frac{\beta_{1}}{\alpha_{1}-\sum_{i=1}^{m-2} \gamma_{i}} \varphi_{q} \\
& \times\left(\frac{1}{r(a)} \int_{a}^{b} h(\tau) f\left(\tau, u(\tau), u^{\prime}(\tau)\right) d \tau\right) \\
& +\frac{1}{\alpha_{1}-\sum_{i=1}^{m-2} \gamma_{i}} \sum_{i=1}^{m-2} \gamma_{i} \\
& \times \int_{a}^{\xi_{i}} \varphi_{q}\left(\frac{1}{r(s)} \int_{s}^{b} h(\tau) f\left(\tau, u(\tau), u^{\prime}(\tau)\right) d \tau\right) d s \\
& \leq \int_{a}^{b} \varphi_{q}\left(\frac{1}{r(a)} \int_{a}^{b} h(\tau) f\left(\tau, u(\tau), u^{\prime}(\tau)\right) d \tau\right) d s \\
& +\frac{\beta_{1}}{\alpha_{1}-\sum_{i=1}^{m-2} \gamma_{i}} \\
& \times \varphi_{q}\left(\frac{1}{r(a)} \int_{a}^{b} h(\tau) f\left(\tau, u(\tau), u^{\prime}(\tau)\right) d \tau\right) \\
& +\frac{1}{\alpha_{1}-\sum_{i=1}^{m-2} \gamma_{i}} \sum_{i=1}^{m-2} \gamma_{i} \\
& \times \int_{a}^{\xi_{i}} \varphi_{q}\left(\frac{1}{r(a)} \int_{a}^{b} h(\tau) f\left(\tau, u(\tau), u^{\prime}(\tau)\right) d \tau\right) d s \\
& \leq \int_{a}^{b} \varphi_{q}\left(\frac{1}{L} \varphi_{p}\left(\frac{m_{1}}{N}\right) \frac{1}{r(a)} \int_{a}^{b} h(\tau) d \tau\right) d s \\
& +\frac{\beta_{1}}{\alpha_{1}-\sum_{i=1}^{m-2} \gamma_{i}} \varphi_{q}\left(\frac{1}{L} \varphi_{p}\left(\frac{m_{1}}{N}\right) \frac{1}{r(a)} \int_{a}^{b} h(\tau) d \tau\right) \\
& +\frac{1}{\alpha_{1}-\sum_{i=1}^{m-2} \gamma_{i}} \sum_{i=1}^{m-2} \gamma_{i} \\
& \times \int_{a}^{\xi_{i}} \varphi_{q}\left(\frac{1}{L} \varphi_{p}\left(\frac{m_{1}}{N}\right) \frac{1}{r(a)} \int_{a}^{b} h(\tau) d \tau\right) d s \\
& \leq \frac{m_{1}}{N}\left(b-a+\frac{\beta_{1}+\sum_{i=1}^{m-2} \gamma_{i}\left(\xi_{i}-a\right)}{\alpha_{1}-\sum_{i=1}^{m-2} \gamma_{i}}\right)=m_{1} .
\end{aligned}
$$

Thus, the conditions $\left(B_{1}\right)-\left(B_{3}\right)$ in Lemma 1 hold, and so the problem $(\mathscr{P})$ has at least three positive solutions $u_{1}, u_{2}$, and $u_{3}$ such that (30) holds.

Similar to the above arguments, we will discuss the problem

$$
\begin{gathered}
\left(r(t) \varphi_{p}\left(u^{\prime}\right)\right)^{\prime}+h(t) f\left(t, u(t), u^{\prime}(t)\right)=0, \quad t \in(a, b), \\
u^{\prime}(a)=0, \quad \alpha_{2} u(b)+\beta_{2} u^{\prime}(b)=\sum_{i=1}^{m-2} \delta_{i} u\left(\xi_{i}\right) .
\end{gathered}
$$

Now, we replace the assumption $\left(A_{1}\right)$ with the following:

$\left(A_{1}^{*}\right) \alpha_{2}, \beta_{2} \in[0, \infty), \delta_{i} \in[0, \infty)$ satisfy $\alpha_{2}>\sum_{i=1}^{m-2} \delta_{i}$, $a<\xi_{1}<\cdots<\xi_{m-2}<b(m>3)$.

We only give the main results of the problem $\left(\mathscr{P}^{*}\right)$, and the proofs are omitted.

Lemma 7. Assume that $\left(A_{1}^{*}\right),\left(A_{2}\right),\left(A_{3}\right)$, and $\left(A_{4}\right)$ hold. Then the problem $\left(\mathscr{P}^{*}\right)$ has a unique solution

$$
\begin{aligned}
u(t)= & \int_{t}^{b} \varphi_{q}\left(\frac{1}{r(s)} \int_{a}^{s} h(\tau) f\left(\tau, u(\tau), u^{\prime}(\tau)\right) d \tau\right) d s \\
& +\frac{1}{\alpha_{2}-\sum_{i=1}^{m-2} \delta_{i}} \\
& \times\left(\beta_{2} \varphi_{q}\left(\frac{1}{r(b)} \int_{a}^{b} h(\tau) f\left(\tau, u(\tau), u^{\prime}(\tau)\right) d \tau\right)\right. \\
& \quad+\sum_{i=1}^{m-2} \delta_{i} \int_{\xi_{i}}^{b} \varphi_{q} \\
& \left.\times\left(\frac{1}{r(s)} \int_{a}^{s} h(\tau) f\left(\tau, u(\tau), u^{\prime}(\tau)\right) d \tau\right) d s\right) .
\end{aligned}
$$

The cone is defined by

$$
\begin{aligned}
& K^{*}=\left\{u \in E: u(t) \geq 0 \text { for } t \in[a, b], u^{\prime \prime}(t) \leq 0,\right. \\
& u^{\prime}(t) \geq 0 \text { for } t \in(a, b), u^{\prime}(a)=0, \\
& \alpha_{2} u(b)+\beta_{2} u^{\prime}(b)=\sum_{i=1}^{m-2} \delta_{i} u\left(\xi_{i}\right) \\
& \text { and } \left.\min _{t \in[\sigma, b-\sigma]} u(t) \geq \tau_{1} \max _{t \in[a, b]} u(t)\right\} \text {. }
\end{aligned}
$$

Lemma 8. Assume that $\left(A_{1}\right)$ holds. If $u \in K^{*}$, then

$$
\min _{t \in[\sigma, b-\sigma]} u(t) \geq \tau_{3}\|u\|_{0}, \quad\|u\|_{0} \leq \tau_{4}\left\|u^{\prime}\right\|_{0},
$$

where $\tau_{3}=\beta_{2} /\left(\beta_{2}+(b-a)\left(\alpha_{2}-\sum_{i=1}^{m-2} \delta_{i}\right)\right)$ and $\tau_{4}=\left(\beta_{2}+\right.$ $\left.\alpha_{2}(b-a)\right) /\left(\alpha_{2}-\sum_{i=1}^{m-2} \delta_{i}\right)$. 
In what follows, we define integral operators $T$ and $L^{*}$, $M^{*}$, and $N^{*}$ :

$$
\begin{aligned}
& (T u)(t) \\
& =\int_{t}^{b} \varphi_{q}\left(\frac{1}{r(s)} \int_{a}^{s} h(\tau) f\left(\tau, u(\tau), u^{\prime}(\tau)\right) d \tau\right) d s \\
& +\frac{1}{\alpha_{2}-\sum_{i=1}^{m-2} \delta_{i}} \\
& \times\left(\beta_{2} \varphi_{q}\left(\frac{1}{r(b)} \int_{a}^{b} h(\tau) f\left(\tau, u(\tau), u^{\prime}(\tau)\right) d \tau\right)+\sum_{i=1}^{m-2} \delta_{i}\right. \\
& \left.\times \int_{\xi_{i}}^{b} \varphi_{q}\left(\frac{1}{r(s)} \int_{a}^{s} h(\tau) f\left(\tau, u(\tau), u^{\prime}(\tau)\right) d \tau\right) d s\right) \\
& L^{*}=\frac{1}{r(b)} \int_{a}^{b} h(\tau) d \tau, \\
& M^{*}=\frac{\alpha_{2}-\sum_{i=1}^{m-2} \delta_{i}}{\beta_{2}}, \\
& N^{*}=b-a+\frac{\beta_{2}+\sum_{i=1}^{m-2} \delta_{i}\left(b-\xi_{i}\right)}{\alpha_{2}-\sum_{i=1}^{m-2} \delta_{i}} .
\end{aligned}
$$

Theorem 9. Suppose that $\left(A_{1}^{*}\right),\left(A_{2}\right),\left(A_{3}\right)$, and $\left(A_{4}\right)$ hold and $f(t, 0,0) \quad \equiv \quad 0$ for a.e. $t \in[a, b]$. If there exist positive numbers $\lambda^{\prime}, m_{1}, m_{2}$, and $m_{4}$ with $m_{1}<m_{2} \leq \tau_{3}^{2} \lambda^{\prime}((b-$ a) $/ r(b))^{-1 /(p-1)} m_{4}$ such that the following conditions are satisfied:

$$
\begin{aligned}
\left(D_{1}\right) & f(t, \mu, v) \leq\left(1 / L^{*}\right) \varphi_{p}\left(m_{4} / L^{*}\right),(t, \mu, v) \in[a, b] \times \\
& {\left[0, \tau_{4} m_{4}\right] \times\left[-m_{4}, m_{4}\right], } \\
\left(D_{2}\right) & f(t, \mu, v)>\left(1 / L^{*}\right) \varphi_{p}\left(m_{2} / M^{*}\right),(t, \mu, v) \in[a, b] \times \\
& {\left[m_{2}, m_{2} \tau_{3}^{-1}\right] \times\left[-m_{4}, m_{4}\right], } \\
\left(D_{3}\right) & f(t, \mu, v) \leq\left(1 / L^{*}\right) \varphi_{p}\left(m_{1} / N^{*}\right),(t, \mu, v) \in[a, b] \times \\
& {\left[0, m_{1}\right] \times\left[-m_{4}, m_{4}\right], }
\end{aligned}
$$

then the problem $\left(\mathscr{P}^{*}\right)$ has at least three positive solutions $u_{1}$, $u_{2}$, and $u_{3}$ satisfying

$$
\begin{gathered}
\max _{t \in[a, b]}\left|u_{i}^{\prime}(t)\right| \leq m_{4} \quad \text { for } i=1,2,3, \\
m_{2}<\min _{t \in[a, b]} u_{1}(t), \quad m_{1}<\max _{t \in[a, b]} u_{2}(t) \\
\text { with } \min _{t \in[a, b]} u_{2}(t)<m_{2}, \\
\max _{t \in[a, b]} u_{3}(t)<m_{1} .
\end{gathered}
$$

From Theorem 6 or Theorem 9, we can see that, when assumptions like $\left(C_{1}\right),\left(C_{2}\right)$, and $\left(C_{3}\right)$ are imposed appropriately on the nonlinear term $f$, we also establish the existence results of an arbitrary odd number of positive solutions of the problem $(\mathscr{P})$ or $\left(\mathscr{P}^{*}\right)$. Here, we only present the form of Theorem 6.
Theorem 10. Suppose that $\left(A_{1}\right)-\left(A_{4}\right)$ hold and $f(t, 0,0) \not \equiv 0$ for a.e. $t \in[a, b]$. If there exist positive numbers $\lambda, m_{1 i}, m_{2 i}$, and $m_{4 i} i=1,2, \ldots$, with

$$
\begin{aligned}
m_{11} & <m_{21} \leq \frac{\tau_{1}^{2} \lambda}{((b-a) / r(a))^{1 /(p-1)}} m_{41}<m_{12}<m_{22} \\
& \leq \frac{\tau_{1}^{2} \lambda}{((b-a) / r(a))^{1 /(p-1)}} m_{42}<\cdots<m_{1 n} \\
& <m_{2 n} \leq \frac{\tau_{1}^{2} \lambda}{((b-a) / r(a))^{1 /(p-1)}} m_{4 n}
\end{aligned}
$$

such that the following conditions are satisfied:

$\left(C_{1}\right) f(t, \mu, \nu) \leq(1 / L) \varphi_{p}\left(m_{4 i}\right),(t, \mu, \nu) \in[a, b] \times$ $\left[0, \tau_{2} m_{4 i}\right] \times\left[-m_{4 i}, m_{4 i}\right]$,

$\left(C_{2}\right) f(t, \mu, \nu)>(1 / L) \varphi_{p}\left(m_{2 i} / \tau_{1} M\right),(t, \mu, \nu) \in[\sigma, b-\sigma] \times$ $\left[m_{2 i}, m_{2 i} \tau_{1}^{-2}\right] \times\left[-m_{4 i}, m_{4 i}\right]$,

$\left(C_{3}\right) f(t, \mu, \nu) \leq(1 / L) \varphi_{p}\left(m_{1} / N\right),(t, \mu, \nu) \in[a, b] \times$ $\left[0, m_{1 i}\right] \times\left[-m_{4 i}, m_{4 i}\right]$,

then the problem $(\mathscr{P})$ has at least $2 n-1$ positive solutions.

\section{Example}

Let $p=3, m=4$, and $\sigma=1 / 4$. Consider the boundary value problem

$$
\begin{gathered}
\left(r(t) \varphi_{p}\left(u^{\prime}\right)\right)^{\prime}+h(t) f\left(t, u(t), u^{\prime}(t)\right)=0, \quad 0<t<1, \\
2 u(0)-u^{\prime}(0)=\frac{1}{2} u\left(\frac{1}{4}\right)+\frac{1}{2} u\left(\frac{1}{2}\right), \quad u^{\prime}(1)=0
\end{gathered}
$$

where

$$
\begin{gathered}
r(t)=t+1, \quad h(t)=\frac{1}{\sqrt{t(1-t)}}, \\
f(t, \mu, v)= \begin{cases}10^{-4} t+2 \mu^{8}+\left(\frac{v}{3 \times 10^{4}}\right)^{3} \times 10^{-5}, & \mu \leq 4, \\
10^{-4} t+131072+\left(\frac{v}{3 \times 10^{4}}\right)^{3} \times 10^{-5}, & \mu>4 .\end{cases}
\end{gathered}
$$

It is easy to check that $\left(A_{1}\right)-\left(A_{4}\right)$ hold. By some calculations, we have $\tau_{1}=1 / 2, \tau_{2}=3, \lambda \approx 1.615, L \approx 3.142$, $M=1$, and $N=2.375$. If we choose $m_{1}=1 / 2, m_{2}=1$, and $m_{4}=3 \times 10^{4}$, then $f(t, \mu, \nu)$ satisfies

$$
\begin{array}{r}
f(t, \mu, \nu) \leq \frac{1}{L} \varphi_{3}\left(m_{4}\right) \approx 2.866 \times 10^{8}, \\
(t, \mu, \nu) \in[0,1] \times\left[0,9 \times 10^{4}\right] \times\left[-3 \times 10^{4}, 3 \times 10^{4}\right], \\
f(t, \mu, \nu)>\frac{1}{L} \varphi_{3}\left(\frac{m_{2}}{\tau_{1} M}\right) \approx 1.274,
\end{array}
$$




$$
\begin{array}{r}
(t, \mu, v) \in\left[\frac{1}{4}, \frac{3}{4}\right] \times[1,4] \times\left[-3 \times 10^{4}, 3 \times 10^{4}\right] \\
f(t, \mu, v) \leq \frac{1}{L} \varphi_{3}\left(\frac{m_{1}}{N}\right) \approx 0.014 \\
(t, \mu, v) \in[0,1] \times\left[0, \frac{1}{2}\right] \times\left[-3 \times 10^{4}, 3 \times 10^{4}\right] .
\end{array}
$$

Thus all assumptions of Theorem 6 hold, and so the problem (44) has at least three positive solutions $u_{1}, u_{2}$, and $u_{3}$ such that $\max _{t \in[0,1]}\left|u_{i}^{\prime}(t)\right| \leq 3 \times 10^{4}$ for $i=$ $1,2,3,1<\min _{(1 / 4) \leq t \leq(3 / 4)} u_{1}(t), 1 / 2<\max _{t \in[0,1]} u_{2}(t)$ with $\min _{(1 / 4) \leq t \leq(3 / 4)} u_{2}(t)<1$, and $\max _{t \in[0,1]} u_{3}(t)<1 / 2$.

\section{References}

[1] R. P. Agarwal and D. O’Regan, "Some new results for singular problems with sign changing nonlinearities," Journal of Computational and Applied Mathematics, vol. 113, no. 1-2, pp. 1-15, 2000.

[2] R. P. Agarwal and D. O'Regan, Singular Differential and Integral Equations with Applications, Kluwer Academic Publishers, 2003.

[3] R. P. Agarwal, D. O’Regan, and P. J. Y. Wong, Positive Solutions of Differential, Difference and Integral Equations, Kluwer Academic Publishers, 1999.

[4] H. Asakawa, "Nonresonant singular two-point boundary value problems," Nonlinear Analysis: Theory, Methods \& Applications, vol. 44, no. 6, pp. 791-809, 2001.

[5] R. Ma and D. O’Regan, "Solvability of singular second order $m$-point boundary value problems," Journal of Mathematical Analysis and Applications, vol. 301, no. 1, pp. 124-134, 2005.

[6] D. O'Regan, Theory of Singular Boundary Value Problem, World Scientific, Hackensack, NJ, USA, 1994.

[7] S. D. Taliaferro, "A nonlinear singular boundary value problem," Nonlinear Analysis: Theory, Methods \& Applications, vol. 3, no. 6, pp. 897-904, 1979.

[8] Y. Wang and W. Ge, "Existence of triple positive solutions for multi-point boundary value problems with a one dimensional p-Laplacian," Computers \& Mathematics with Applications, vol. 54, no. 6, pp. 793-807, 2007.

[9] Q. Yao, "Existence and iteration of $n$ symmetric positive solutions for a singular two-point boundary value problem," Computers \& Mathematics with Applications, vol. 47, no. 8-9, pp. 1195-1200, 2004.

[10] Y. Zhang, "Positive solutions of singular sublinear EmdenFowler boundary value problems," Journal of Mathematical Analysis and Applications, vol. 185, no. 1, pp. 215-222, 1994.

[11] Y. Zhang, "A multiplicity result for a singular generalized Sturm-Liouville boundary value problem," Mathematical and Computer Modelling, vol. 50, no. 1-2, pp. 132-140, 2009.

[12] N. Anderson and A. M. Arthurs, "Analytical bounding functions for diffusion problems with Michaelis-Menten kinetics," Bulletin of Mathematical Biology, vol. 47, no. 1, pp. 145-153, 1985.

[13] D. Aronson, M. G. Crandall, and L. A. Peletier, "Stabilization of solutions of a degenerate nonlinear diffusion problem," Nonlinear Analysis: Theory, Methods \& Applications, vol. 6, no. 10, pp. 1001-1022, 1982.

[14] J. W. Bebernes and D. R. Kassoy, "A mathematical analysis of blowup for thermal reactions-the spatially nonhomogeneous case," SIAM Journal on Applied Mathematics, vol. 40, no. 3, pp. 476-484, 1981

[15] A. Nachman and A. Callegari, "A nonlinear singular boundary value problem in the theory of pseudoplastic fluids," SIAM Journal on Applied Mathematics, vol. 38, no. 2, pp. 275-281, 1980.

[16] H. Feng and W. Ge, "Existence of three positive solutions for $m$-point boundary-value problems with one-dimensional $p$ Laplacian," Nonlinear Analysis: Theory, Methods \& Applications, vol. 68, no. 7, pp. 2017-2026, 2008

[17] S. Lin, "Oxygen diffusion in a spherical cell with nonlinear oxygen uptake kinetics," Journal of Theoretical Biology, vol. 60, pp. 449-457, 1976.

[18] C. P. Gupta, "A generalized multi-point boundary value problem for second order ordinary differential equations," Applied Mathematics and Computation, vol. 89, no. 1-3, pp. 133-146, 1998.

[19] L. Kong and Q. Kong, "Multi-point boundary value problem of second order differential equations," Nonlinear Analysis: Theory, Methods \& Applications, vol. 58, pp. 909-931, 2004.

[20] N. Kosmatov, "Symmetric solutions of a multi-point boundary value problem," Journal of Mathematical Analysis and Applications, vol. 309, no. 1, pp. 25-36, 2005.

[21] Y.-W. Zhang and H.-R. Sun, "Three positive solutions for a generalized Sturm-Liouville multipoint BVP with dependence on the first order derivative," Dynamic Systems and Applications, vol. 17, no. 2, pp. 313-324, 2008.

[22] D.-X. Ma, Z.-J. Du, and W.-G. Ge, "Existence and iteration of monotone positive solutions for multipoint boundary value problem with $p$-Laplacian operator," Computers \& Mathematics with Applications, vol. 50, no. 5-6, pp. 729-739, 2005.

[23] W.-S. Cheung and J. Ren, "Twin positive solutions for quasilinear multi-point boundary value problems," Nonlinear Analysis: Theory, Methods \& Applications, vol. 62, no. 1, pp. 167-177, 2005.

[24] R. Avery and J. Henderson, "Existence of three positive pseudosymmetric solutions for a one-dimensional $p$-Laplacian," Journal of Mathematical Analysis and Applications, vol. 277, no. 2, pp. 395-404, 2003.

[25] Y. Zhang, "Existence and multiplicity results for a class of generalized one-dimensional $p$-Laplacian problem," Nonlinear Analysis: Theory, Methods \& Applications, vol. 72, no. 2, pp. 748756, 2010.

[26] R. I. Avery and A. C. Peterson, "Three positive fixed points of nonlinear operators on ordered Banach spaces," Computers \& Mathematics with Applications, vol. 42, no. 3-5, pp. 313-322, 2001. 


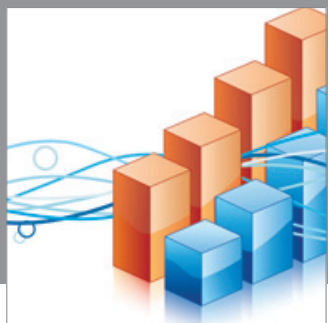

Advances in

Operations Research

mansans

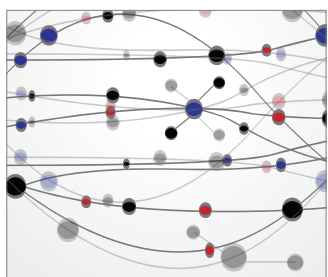

The Scientific World Journal
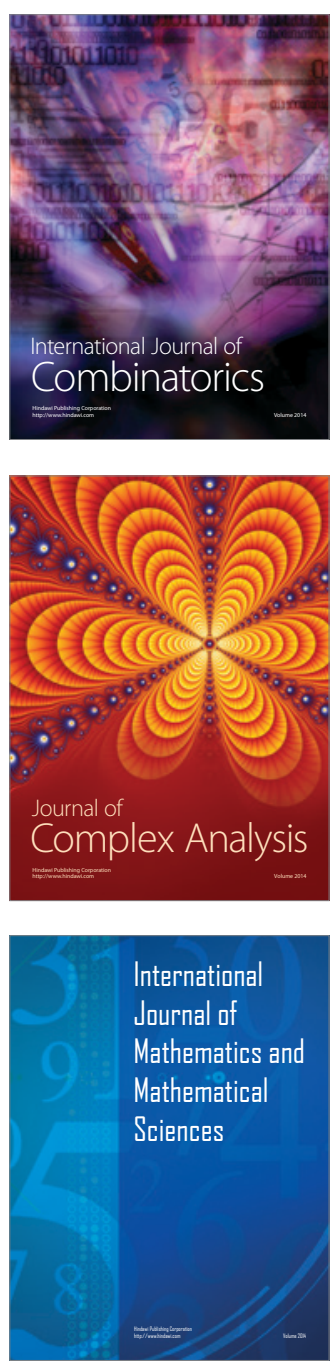
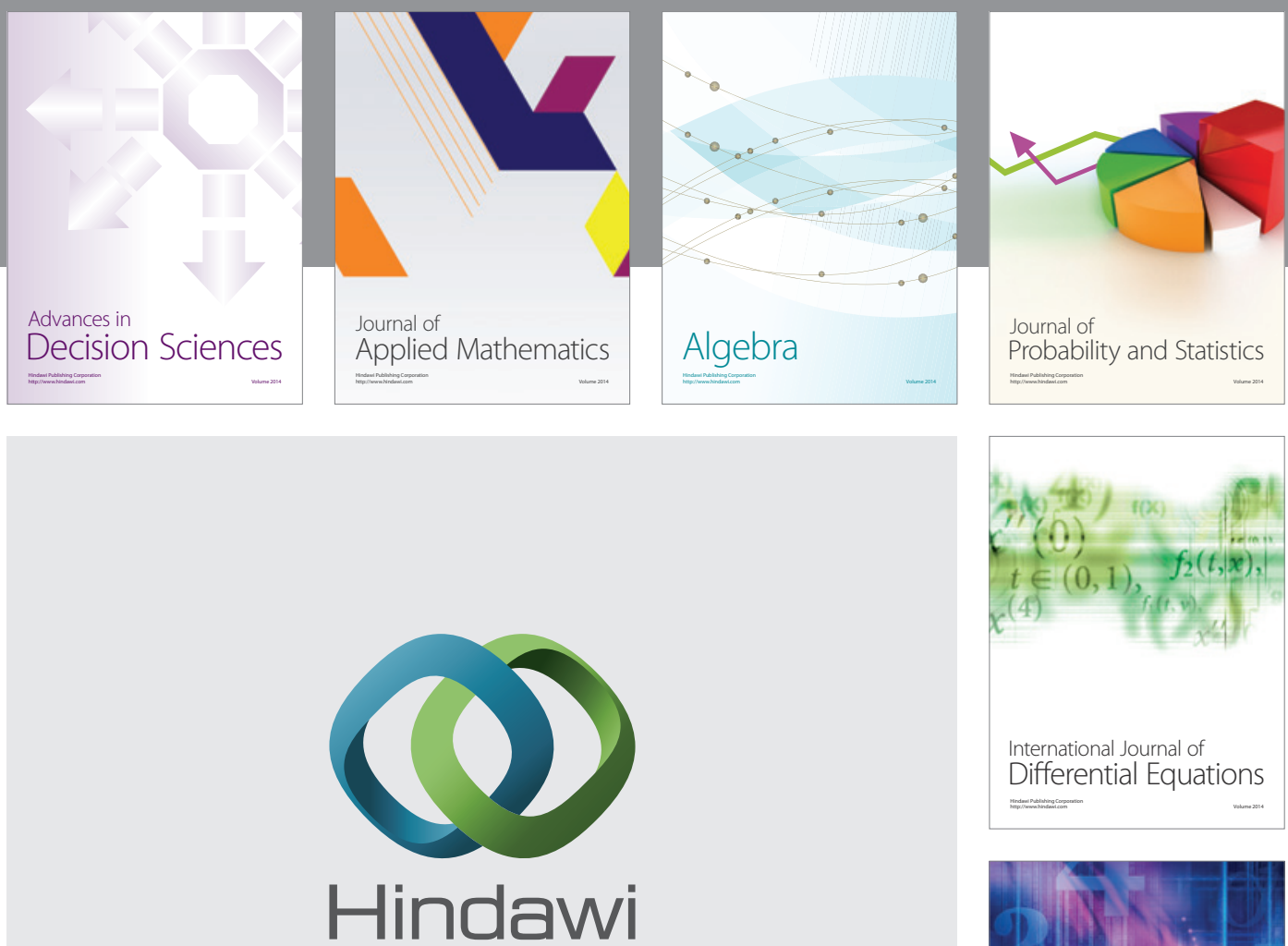

Submit your manuscripts at http://www.hindawi.com
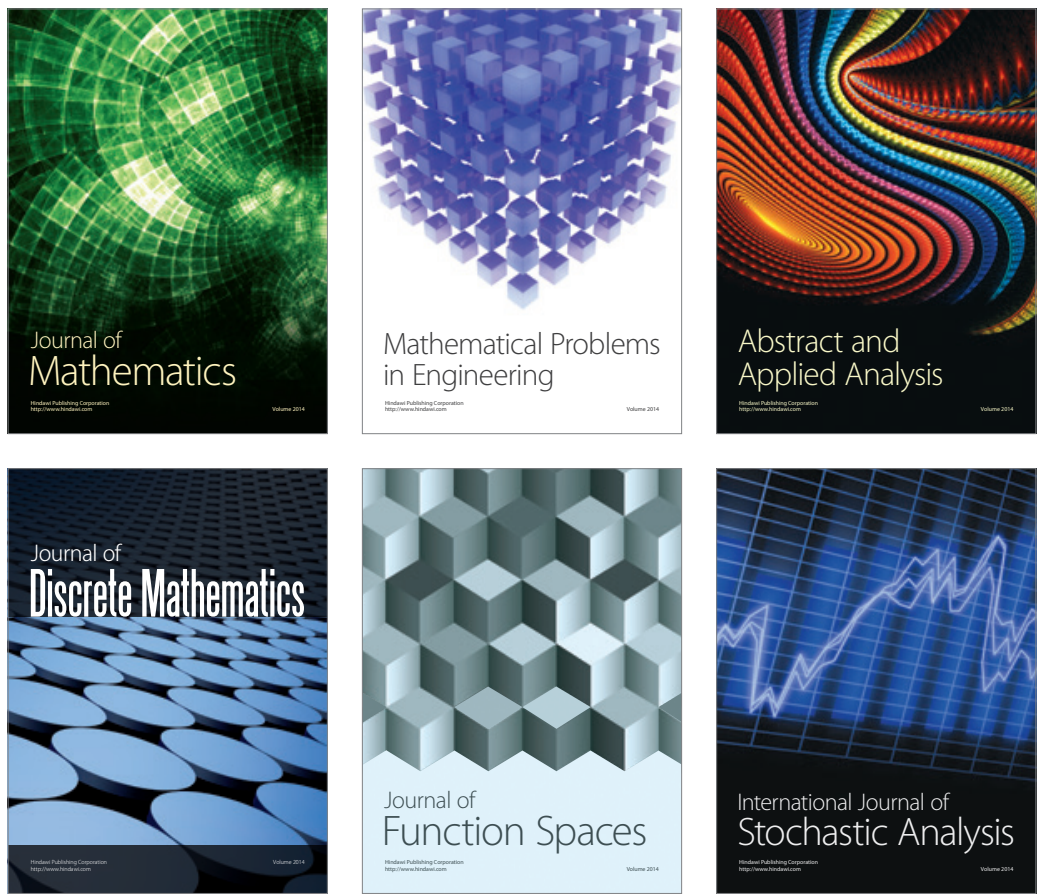

Journal of

Function Spaces

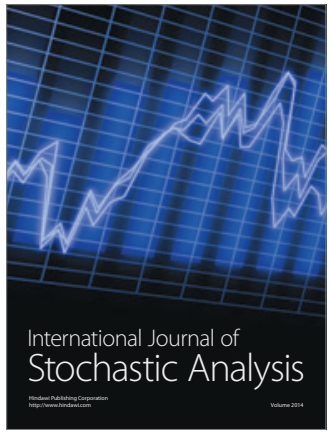

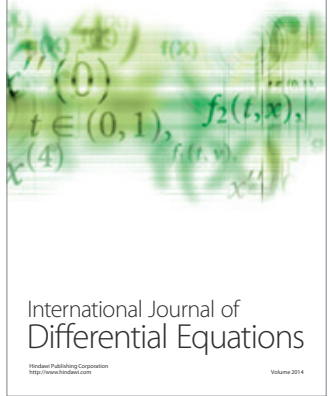
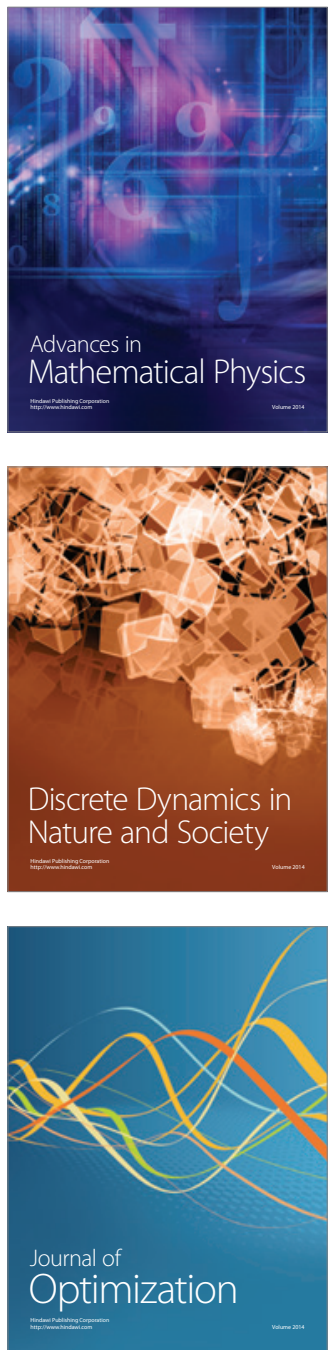